Article

\title{
Bis(6-Diphenylphosphinoacenaphth-5-yl)Telluride as a Ligand toward Manganese and Rhenium Carbonyls
}

\author{
Truong Giang Do, Emanuel Hupf ${ }^{(}$, Enno Lork and Jens Beckmann *(D) \\ Institut für Anorganische Chemie und Kristallographie, Universität Bremen, Leobener Straße 7, \\ 28359 Bremen, Germany; do_giang@yahoo.de (T.G.D.); hupf@uni-bremen.de (E.H.); \\ enno.lork@uni-bremen.de (E.L.) \\ * Correspondence: j.beckmann@uni-bremen.de
}

Academic Editor: J. Derek Woollins

Received: 4 October 2018; Accepted: 26 October 2018; Published: 29 October 2018

Abstract: The reaction of the previously known bis(6-diphenylphosphinoacenaphthyl-5-)telluride (6- $\left.\mathrm{Ph}_{2} \mathrm{P}-\mathrm{Ace}-5-\right)_{2} \mathrm{Te}(\mathrm{IV})$ with $(\mathrm{CO})_{5} \mathrm{ReCl}$ and $(\mathrm{CO})_{5} \mathrm{MnBr}$ proceeded with the liberation of $\mathrm{CO}$ and provided fac-(6- $\left.\mathrm{Ph}_{2} \mathrm{P}-\mathrm{Ace}-5-\right)_{2} \mathrm{TeM}(\mathrm{X})(\mathrm{CO})_{3}(f a c-1$ : $\mathrm{M}=\mathrm{Re}, \mathrm{X}=\mathrm{Cl}$; fac-2: $\mathrm{M}=\mathrm{Mn}, \mathrm{X}=\mathrm{Br})$, in which IV acts as bidentate ligand. In solution, $f a c-\mathbf{1}$ and $f a c-2$ are engaged in a reversible equilibrium with mer-(6- $\left.\mathrm{Ph}_{2} \mathrm{P}-\mathrm{Ace}-5-\right)_{2} \mathrm{TeM}(\mathrm{X})(\mathrm{CO})_{3}$ (mer-1: $\mathrm{M}=\mathrm{Re}, \mathrm{X}=\mathrm{Cl}$; mer-2: $\left.\mathrm{M}=\mathrm{Mn}, \mathrm{X}=\mathrm{Br}\right)$. Unlike fac-1, fac-2 is prone to release another equivalent of $\mathrm{CO}$ to give $\left(6-\mathrm{Ph}_{2} \mathrm{P}-\mathrm{Ace}-5-\right)_{2} \mathrm{TeMn}(\mathrm{Br})(\mathrm{CO})_{2}(3)$, in which IV serves as tridentate ligand.

Keywords: ligand design; transition metal complex; tellurium; manganese; rhenium

\section{Introduction}

Transition metal complexes composed of multidentate ligands based upon tellurium are far less explored than those of sulfur and selenium, which might be due to the lack of easily available ligands containing tellurium, as well as the historical misconception that organotellurium compounds were extremely malodorous and toxic [1-8]. Up to the 1970s, studies of coordination chemistry were mostly restricted to monodentate ligands. The first bidentate telluroether ligand I (Te, P-type) was described by Gysling and Luss in 1984 [9], whereas the first tridentate telluroethers II (N,Te,N-type), and III (P,Te,P-type) were reported by Singh et al. [10] as well as Lin and Gabbaï (Scheme 1) [11].<smiles>c1ccc([Te]c2ccccc2P(c2ccccc2)c2ccccc2)cc1</smiles><smiles>c1ccc(P(c2ccccc2)c2ccccc2P(c2ccccc2)c2ccccc2)cc1</smiles><smiles>CN(C)Cc1cccc2cccc(Oc3cccc4cccc(CN(C)C)c34)c12</smiles><smiles>c1ccc([Te]c2ccc3c4c(ccc(P(c5ccccc5)c5ccccc5)c24)CC3)cc1</smiles>

II

IV

Scheme 1. Bidentate and tridentate telluroether ligands I-IV. 
In preceding work, we reported on the synthesis of bis(6-diphenylphosphinoacenaphth-5-yl) telluride (IV) [12], which also holds potential as a tridentate telluroether ligand. Herein, we describe the reaction of IV with $(\mathrm{CO})_{5} \mathrm{MnBr}$ and $(\mathrm{CO})_{5} \mathrm{ReCl}$, giving rise to the formation of three octahedral transition metal carbonyl complexes with this ligand.

\section{Results and Discussion}

The reaction of bis(6-diphenylphosphinoacenaphth-5-yl)telluride (6- $\mathrm{Ph}_{2} \mathrm{P}$-Ace-5-) ${ }_{2} \mathrm{Te}$ (IV) with $(\mathrm{CO})_{5} \mathrm{ReCl}$ in THF at $65^{\circ}$ for 7 days afforded an 1:1 isomeric mixture of the complex fac/mer-(6-Ph $\left.{ }_{2} \mathrm{P}-\mathrm{Ace}-5-\right)_{2} \mathrm{TeRe}(\mathrm{Cl})(\mathrm{CO})_{3}($ fac-1/mer-1), which slowly interconvert in solution. The progress of the reaction was followed by ${ }^{31} \mathrm{P}$ NMR spectroscopy. Upon cooling to r.t. solely fac-1 precipitates from the reaction mixture and was isolated in $45 \%$ isolated yield (Scheme 2).

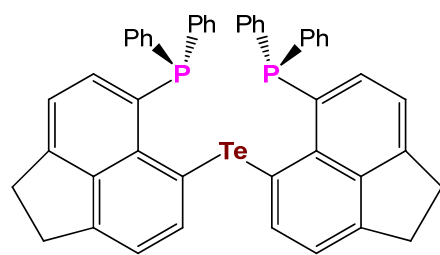

IV
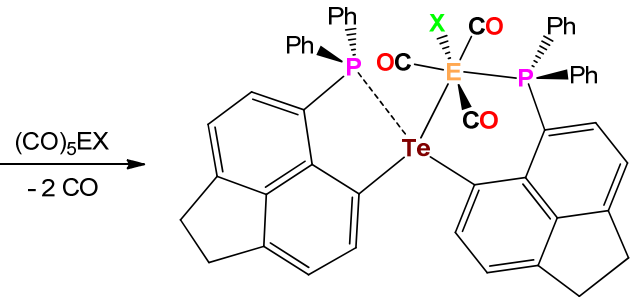

fac-1, $\mathrm{E}=\mathrm{Re}, \mathrm{X}=\mathrm{Cl}$ fac-2, $\mathrm{E}=\mathrm{Mn}, \mathrm{X}=\mathrm{Br}$<smiles>CC(C)=O</smiles>

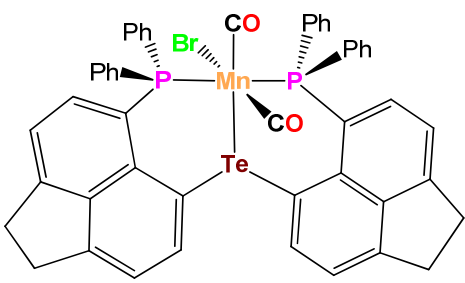

3

Scheme 2. Synthesis of the complexes fac-1, fac-2, and 3.

The analogous reaction of $\mathbf{I V}$ with $(\mathrm{CO})_{5} \mathrm{MnBr}$ in THF at $65^{\circ}$ occurred within $32 \mathrm{~h}$ and gave rise to the isomeric mixture of $f a c / m e r-\left(6-\mathrm{Ph}_{2} \mathrm{P}-\mathrm{Ace}-5-\right)_{2} \mathrm{TeMe}(\mathrm{Br})(\mathrm{CO})_{3}(f a c-2 / m e r-2)$ and as was already observed for the Te-Re complex fac-1, fac-2 precipitates solely from the reaction mixture upon cooling to r.t. and was isolated in $47 \%$ yield (Scheme 2). In both complexes fac-1 and fac-2, the telluroether IV serves as bidentate ligand. In the solid-state, $f a c-\mathbf{1}$ and $f a c-\mathbf{2}$ are reasonably stable toward moist air and show no signs of decomposition even after prolonged times of storage. Unfortunately, both complexes show only poor solubility in the most common solvents used for NMR spectroscopy. In $\mathrm{CDCl}_{3}$ solution, both $f a c-\mathbf{1}$ and $f a c-2$ are in equilibrium with their related meridional complexes mer-1 and mer-2, which can be inferred by ${ }^{31} \mathrm{P}-\mathrm{NMR}$ spectroscopy (Scheme 3). The ${ }^{31} \mathrm{P}-\mathrm{NMR}$ spectrum of a freshly prepared solution of $\mathrm{fac}-\mathbf{1}$ in $\mathrm{CDCl}_{3}$, shows almost exclusively two chemical shifts at $\delta=-0.9$ and $-25.0 \mathrm{ppm}$ of equal intensity, which are assigned to the $\mathrm{P}$ atom that coordinates to the Re atom and the $\mathrm{P}$ atom that engages in interaction with the Te atom (the assignment is based upon the comparison with $f a c-\left[\operatorname{Re}(\mathrm{Cl})(\mathrm{CO})_{3}\left(\mathrm{PPh}_{2} \mathrm{C}_{10} \mathrm{H}_{6} \mathrm{PPh}_{2}\right)\right][13],\left(\left[\left(\mathrm{Ph}_{2} \mathrm{P}\left(\mathrm{Me}_{2} \mathrm{pz}\right)_{2}\right) \operatorname{Re}(\mathrm{CO})_{3} \mathrm{Br}\right]\right.$ and $\left[\left(\mathrm{Ph}_{2} \mathrm{P}\left(\mathrm{Me}_{2} \mathrm{pz}\right)\right) \operatorname{Re}(\mathrm{CO})_{4} \mathrm{Br}(\mathrm{pz}=\right.$ pyrazole) [14]). However, the formation of mer-1 can already be detected even from the freshly prepared solution of fac-1, showing chemical shifts at $\delta=14.7$ and $-26.4 \mathrm{ppm}$, which increase in intensity over time (Figure 1). It should be noted that the chemical shifts of $f a c-\mathbf{1}$ consist of singlets, whereas the doublets were observed for mer-1 with a coupling constant of $J\left({ }^{31} \mathrm{P}-{ }^{31} \mathrm{P}\right)=11.6 \mathrm{~Hz}$. In contrast, the Te-Mn complex 2 shows significantly faster formation of 
mer-2 $\left(\delta\left({ }^{31} \mathrm{P}\right)=50.0\right.$ and $\left.-28.0 \mathrm{ppm}\right)$ from a freshly prepared solution of fac-1 $\left(\delta\left({ }^{31} \mathrm{P}\right)=36.2\right.$ and $-25.6 \mathrm{ppm}$, Figure S2d).
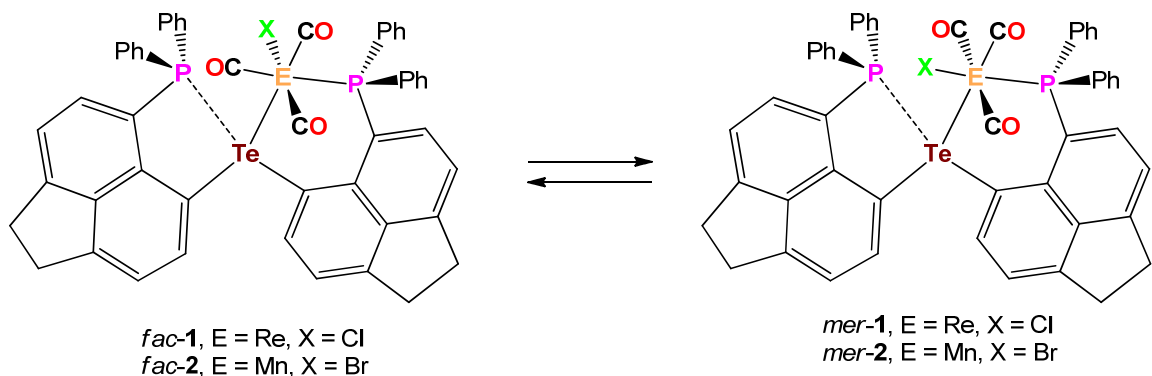

Scheme 3. Dynamic equilibrium between fac-1 and mer-1 as well as fac-2 and mer-2.

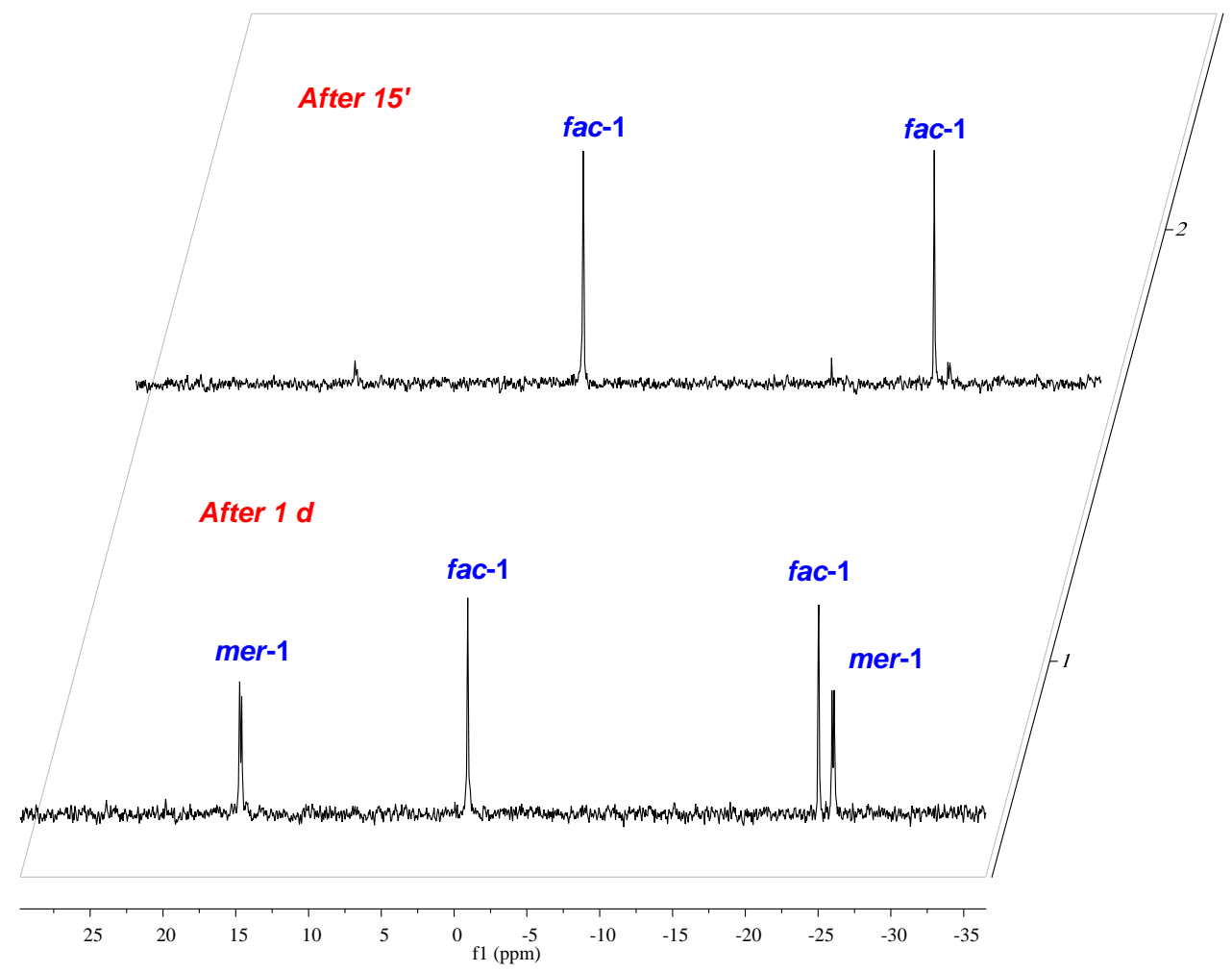

Figure 1. Equilibration between $f a c-\mathbf{1}$ and $m e r-\mathbf{1}$ in the $\mathrm{CDCl}_{3}$ solution.

When the reaction of $\mathbf{I V}$ with $(\mathrm{CO})_{5} \mathrm{MnBr}$ was repeated with a longer reaction time of 7 days, a different product, namely (6- $\left.\mathrm{Ph}_{2} \mathrm{P}-\mathrm{Ace}-5-\right)_{2} \mathrm{TeMn}(\mathrm{Br})(\mathrm{CO})_{2}$ (3) formed, which was obtained in $40 \%$ isolated yield (Scheme 2). The formation of $\mathbf{3}$ can be rationalized by the liberation of $\mathrm{CO}$ from $\mathrm{fac}-\mathbf{2}$ and/or mer-2. In 3, the telluroether serves as tridentate ligand. Notably, a similar reactivity of fac-1 was not observed. The solubility of $\mathbf{3}$ in the most common solvents is slightly higher than those of fac-1 and fac-2, which allowed the acquisition of full set of NMR data. The ${ }^{31} \mathrm{P}-\mathrm{NMR}$ spectrum $\left(\mathrm{CDCl}_{3}\right)$ of 3 shows a broad signal at $\delta=59.7 \mathrm{ppm}\left(\omega_{1 / 2}=110 \mathrm{~Hz}\right)$, whereas the ${ }^{125} \mathrm{Te}-\mathrm{NMR}$ spectrum exhibits a singlet at $\delta=753.1 \mathrm{pm}$ that is slightly high-field shifted in comparison to IV ( $\delta=704.4 \mathrm{ppm})$ [12].

The molecular structures of $f a c-1, f a c-2$, and $\mathbf{3}$ are shown in Figures 2-4. Selected bond parameters are collected in Table 1. The spatial arrangement of the Re and Mn atoms is octahedral. In fac-1 and fac-2, only one $\mathrm{P}$ atom coordinates to the Re and Mn atoms, whereas the other $\mathrm{P}$ atoms engage in a through-space interactions with the Te atoms. Consistent with -the covalent radii of $\mathrm{Mn}$ and Re, the Mn-Te bond length of fac-2 (2.599(1) $\AA$ ) and 3 (2.546(1) $\AA$ ) are smaller than the Re-Te bond length of 
fac-1 (2.733(1) $\AA$ ). The Mn-Te distances are in between values reported for $\left[\mathrm{Mn}(\mathrm{CO})_{4} \mathrm{TePh}\right]_{2}$ (mean 2.674(2) ̊̊) [15-17] and [Cp' $(\mathrm{CO})_{2} \mathrm{Mn}_{2} \mathrm{TeMes}^{+}(2.44 \AA)$ [18].

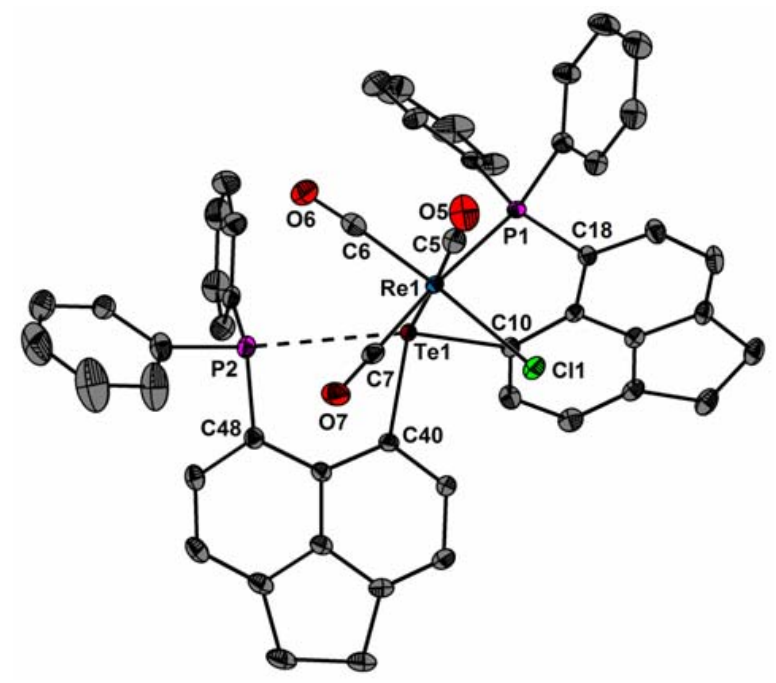

Figure 2. Molecular structure of fac-1 showing 50\% probability ellipsoids and the crystallographic numbering scheme.

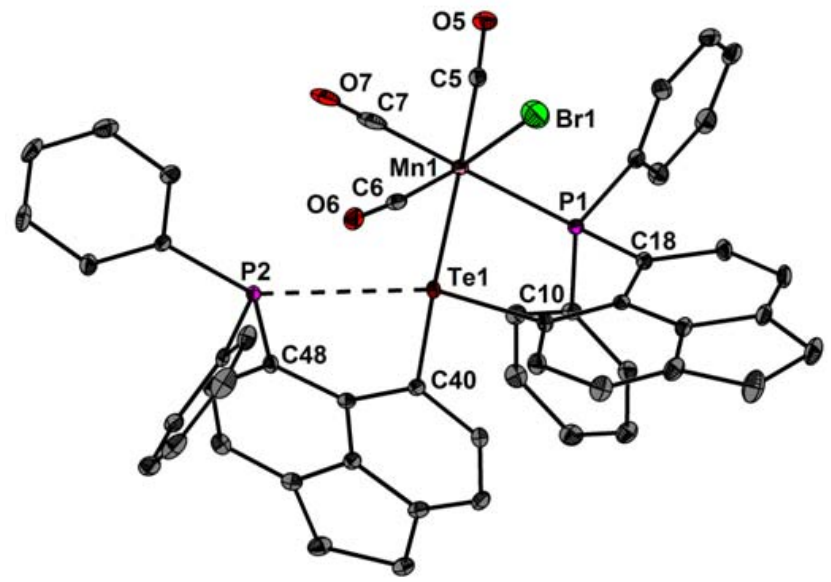

Figure 3. Molecular structure of fac-2 showing 50\% probability ellipsoids and the crystallographic numbering scheme.

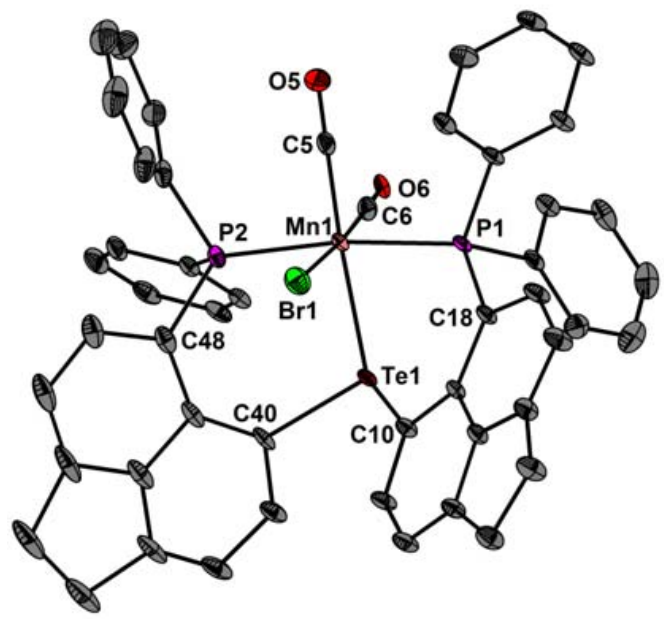

Figure 4. Molecular structure of 3 showing 50\% probability ellipsoids and the crystallographic numbering scheme. 
Table 1. Experimental interatomic distances $[\AA]$ and angles $\left[^{\circ}\right]$ of $f a c-\mathbf{1}, f a c-2$, and 3 .

\begin{tabular}{|c|c|c|c|}
\hline & fac-1 $(\mathbf{M}=\mathbf{R e})$ & fac-2 (M = Mn) & $3(\mathbf{M}=\mathbf{M n})$ \\
\hline \multicolumn{4}{|c|}{ Bond Lengths and Angles } \\
\hline $\mathrm{M}(1)-\mathrm{Te}(1)$ & $2.733(1)$ & $2.599(1)$ & $2.546(1)$ \\
\hline $\mathrm{M}(1)-\mathrm{P}(1)$ & $2.466(1)$ & $2.343(1)$ & $2.293(1)$ \\
\hline $\mathrm{M}(1)-\mathrm{P}(2)$ & - & - & $2.277(1)$ \\
\hline $\mathrm{M}(1)-\mathrm{X}(1)$ & $2.487(1)$ & $2.498(1)$ & $2.540(1)$ \\
\hline $\mathrm{M}(1)-\mathrm{C}(5)$ & $1.930(2)$ & $1.819(5)$ & $1.800(4)$ \\
\hline $\mathrm{M}(1)-\mathrm{C}(6)$ & $1.930(2)$ & $1.817(6)$ & $1.811(4)$ \\
\hline $\mathrm{M}(1)-\mathrm{C}(7)$ & $1.951(2)$ & $1.915(7)$ & - \\
\hline $\mathrm{C}(5)-\mathrm{O}(5)$ & $1.142(2)$ & $1.127(7)$ & $1.146(6)$ \\
\hline $\mathrm{C}(6)-\mathrm{O}(6)$ & $1.121(2)$ & $1.108(7)$ & $1.037(5)$ \\
\hline $\mathrm{C}(7)-\mathrm{O}(7)$ & $1.141(2)$ & $0.993(8)^{a}$ & - \\
\hline $\mathrm{P}(1)-\mathrm{M}(1)-\mathrm{P}(2)$ & - & - & $170.8(1)$ \\
\hline $\mathrm{P}(1)-\mathrm{M}(1)-\mathrm{C}(7)$ & $171.9(1)$ & $171.2(2)$ & - \\
\hline $\mathrm{X}(1)-\mathrm{M}(1)-\mathrm{C}(6)$ & $176.2(1)$ & $173.9(2)$ & $176.3(1)$ \\
\hline $\mathrm{Te}(1)-\mathrm{M}(1)-\mathrm{C}(5)$ & $174.0(1)$ & $175.9(2)$ & $175.6(1)$ \\
\hline \multicolumn{4}{|c|}{ peri Region distances } \\
\hline $\mathrm{P}(1) \cdots \mathrm{Te}(1)$ & $3.363(1)$ & $3.466(1)$ & $3.247(2)$ \\
\hline $\mathrm{P}(1) \cdots \mathrm{Te}(2)$ & $3.111(1)$ & $3.141(1)$ & $3.429(1)$ \\
\hline \multicolumn{4}{|c|}{ peri Region bond Angles } \\
\hline $\mathrm{P}(1)-\mathrm{C}(18)-\mathrm{C}(19) / \mathrm{P}(2)-\mathrm{C}(48)-\mathrm{C}(49)$ & $122.9(1) / 119.5(1)$ & $124.6(3) / 118.8(3)$ & $124.3(3) / 121.0(3)$ \\
\hline$C(10)-C(19)-C(18) / C(40)-C(49)-C(48)$ & 130.2(1)/129.0(1) & $129.8(4) / 129.9(4)$ & $130.3(4) / 129.8(4)$ \\
\hline $\mathrm{Te}(1)-\mathrm{C}(10)-\mathrm{C}(19) / \mathrm{Te}(1)-\mathrm{C}(40)-\mathrm{C}(49)$ & $127.6(1) / 122.0(1)$ & $129.6(3) / 124.1(3)$ & $123.2(3) / 126.1(3)$ \\
\hline$\sum$ of bay angles & $380.7(3) / 370.5(3)$ & $380.0(10) / 372.8(1)$ & $377.8(1) / 376.9(1)$ \\
\hline Splay angle ${ }^{\mathrm{b}}$ & $20.7(3) / 10.5(3)$ & $20(10) / 12.8(1)$ & $17.8(1) / 17.4(1)$ \\
\hline \multicolumn{4}{|c|}{ Out-of-Plane Displacement } \\
\hline $\mathrm{Te}(1)^{\mathrm{c}}$ & $0.198(1) /-0.626(1)$ & $-0.191(1) /-0.390(1)$ & $0.0899(3) /-0.666(1)$ \\
\hline $\mathrm{P}(1)$ & $-0.203(1)$ & $0.097(1)$ & $-0.160(1)$ \\
\hline $\mathrm{P}(2)$ & $0.278(1)$ & $0.263(1)$ & $0.543(1)$ \\
\hline \multicolumn{4}{|c|}{ Central Acenaphthene Ring Torsion Angles } \\
\hline C:(13)-(14)-(19)-(18)/(43)-(44)-(49)-(48) & $175.5(1) / 177.9(1)$ & $-179.3(6) /-173.3(5)$ & $179.4(4) / 169.4(5)$ \\
\hline C:(15)-(14)-(19)-(10)/(45)-(44)-(49)-(40) & 178.7(1)/176.2(1) & $178.5(5) /-177.5(5)$ & $179.4(4) / 178.6(5)$ \\
\hline
\end{tabular}

Likewise, the Mn-P bond length of fac-2 (2.343(1) $\AA$ ) and 3 (2.341(1) $\AA$ ) is smaller than the Re-P bond length of 1 (2.466(1) $\AA$ ). The average Mn-P distances are comparable with those of

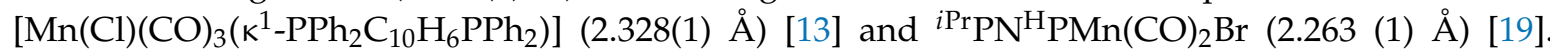
The $\operatorname{Re}(1)-\mathrm{C}(5) / \mathrm{C}(6)$ bond distances of $f a c-1(2 \times 1.930(2) \AA)$ and the $\mathrm{Mn}(1)-\mathrm{C}(5) / \mathrm{C}(6)$ bond distances of $f a c-2(1.817(6), 1.819(5) \AA)$ are very similar to those of $\left[\operatorname{ReCl}(\mathrm{CO})_{3}\left(\mathrm{PPh}_{2} \mathrm{C}_{10} \mathrm{H}_{6} \mathrm{PPh}_{2}\right)\right]$ (mean 1.952(16) $\AA)[13]$ and $f a c-\left[\mathrm{MnCl}(\mathrm{CO})_{3}\left(\mathrm{PPh}_{2} \mathrm{C}_{10} \mathrm{H}_{6} \mathrm{PPh}_{2}\right)\right]$ (mean 1.815(2) $\AA$ ) [20]. Due to the trans-effect of the $\mathrm{P}$ atom, the $\operatorname{Re}(1)-\mathrm{C}(7)$ bond distance of $f a c-1$ (1.951(2) $\AA$ ) and the $\mathrm{Mn}(1)-\mathrm{C}(7)$ distance of fac-2 (1.915(7) $\AA$ ) are slightly longer. The $M n(1)-C(5)$ and $M n(1)-C(6)$ bond lengths of 3 (1.800(4), $1.811(5) \AA)$ are in average very similar than that of $f a c-\left[\mathrm{Mn}(\mathrm{CO})_{3}(\mathrm{dppbz}) \mathrm{Br}\right]($ mean $1.807(4) \AA$; $\mathrm{dppbz}=1,2$-(diphenylphosphino)benzene) [21]. In the carbonyl region of the IR spectrum of fac-1 three strong absorption bands at $\widetilde{v}=2018,1928$, and $1882 \mathrm{~cm}^{-1}$ are visible, respectively, consistent with a facial arrangement of three carbonyl groups [12,22]. The IR spectrum of 3 reveals asymmetric and symmetric stretching vibrations at $\widetilde{v}=1936$ and $1852 \mathrm{~cm}^{-1}$, which compare well with those of ('PrPONOP)Mn(CO) ${ }_{2} \operatorname{Br}\left(1866,1946 \mathrm{~cm}^{-1}\right)$ and ${ }^{\mathrm{i}} \operatorname{PrPNHPMn}(\mathrm{CO})_{2} \mathrm{Br}\left(1824,1916 \mathrm{~cm}^{-1}\right)$ [19].

\section{Conclusions}

The present study demonstrates the ability of bis(6-diphenylphosphinoacenaphthyl-5-)telluride $\left(6-\mathrm{Ph}_{2} \mathrm{P}-\text { Ace-5- }\right)_{2} \mathrm{Te}$ (IV) [12] to act as a bidentate and tridentate ligand toward transition metals 
carbonyls. The reaction of IV with $(\mathrm{CO})_{5} \mathrm{ReCl}$ and $(\mathrm{CO})_{5} \mathrm{MnBr}$ provided three complexes, namely fac-(6-Ph $\left.{ }_{2} \mathrm{P}-\mathrm{Ace}-5-\right)_{2} \mathrm{TeM}(\mathrm{X})(\mathrm{CO})_{3}($ fac-1: $\mathrm{M}=\mathrm{Re}, \mathrm{X}=\mathrm{Cl} ;$ fac-2: $\mathrm{M}=\mathrm{Mn}, \mathrm{X}=\mathrm{Br}$ ) and $\left(6-\mathrm{Ph}_{2} \mathrm{P} \text {-Ace-5- }\right)_{2} \mathrm{TeMn}(\mathrm{Br})(\mathrm{CO})_{2}$ (3). In solution, the facial complexes, fac- $\mathbf{1}$ and $f a c-2$, are engaged in a reversible equilibrium with their meridional complexes, mer- $\left(6-\mathrm{Ph}_{2} \mathrm{P}-\mathrm{Ace}-5-\right)_{2} \mathrm{TeM}(\mathrm{X})(\mathrm{CO})_{3}($ mer-1: $\mathrm{M}=\mathrm{Re}, \mathrm{X}=\mathrm{Cl}$; $m e r-2: \mathrm{M}=\mathrm{Mn}, \mathrm{X}=\mathrm{Br}$ ).

\section{Experimental Section}

General. Reagents were obtained commercially (Sigma-Aldrich, Taufkirchen, Germany) and were used as received. Dry solvents were collected from an SPS800 mBraun solvent system. Bis(6-diphenylacenaphth-5-yl)telluride, (6- $\left.\mathrm{Ph}_{2} \mathrm{P}-\mathrm{Ace}-5-\right)_{2} \mathrm{Te}$ (IV) was prepared according to literature procedures [12]. ${ }^{1} \mathrm{H}-,{ }^{13} \mathrm{C}-,{ }^{31} \mathrm{P}-$, and ${ }^{125} \mathrm{Te}-\mathrm{NMR}$ spectra were recorded at room temperature using a Bruker Avance-360 and a Bruker Avance-200 spectrometer and are referenced to tetramethylsilane $\left({ }^{1} \mathrm{H},{ }^{13} \mathrm{C}\right)$, phosphoric acid $\left(85 \%\right.$ in water) $\left({ }^{31} \mathrm{P}\right)$, and dimethyltelluride $\left({ }^{125} \mathrm{Te}\right)$. Chemical shifts are reported in parts per million (ppm), and coupling constants $(J)$ are given in Hertz (Hz). The FTIR spectra were recorded on Thermo Scientific Nicolet ${ }^{\mathrm{TM}}$ iS10. The ESI-MS spectra were obtained with a Bruker Esquire-LC MS. Dichloromethane/acetonitrile solutions $\left(c=1 \times 10^{-6} \mathrm{~mol} \mathrm{~L}^{-1}\right)$ were injected directly into the spectrometer at a flow rate of $3 \mu \mathrm{L} \mathrm{min}{ }^{-1}$. Nitrogen was used both as a drying gas and for nebulization with flow rates of approximately $5 \mathrm{~L} \mathrm{~min}^{-1}$ and a pressure of 5 psi. Pressure in the mass analyzer region was usually about $1 \times 10^{-5}$ mbar. Spectra were collected for $1 \mathrm{~min}$ and averaged. The nozzle-skimmer voltage was adjusted individually for each measurement.

Synthesis of (6-Ph $\left.{ }_{2} \mathrm{P}-\mathrm{Ace}-5-\right)_{2} \mathrm{Te} \operatorname{Re}(\mathrm{CO})_{3} \mathrm{Cl}(\mathrm{fac}-1): \operatorname{Re}(\mathrm{CO}){ }_{5} \mathrm{Cl}(9.20 \mathrm{mg}, 25.4 \mu \mathrm{mol})$ was added to a solution of (6-Ph ${ }_{2} \mathrm{P}-$ Ace- $\left.-5-\right)_{2} \mathrm{Te}(20.0 \mathrm{mg}, 24.9 \mu \mathrm{mol})$ in THF $(5 \mathrm{~mL})$. The mixture was continually stirred at $65^{\circ} \mathrm{C}$ for $7 \mathrm{~d}$. The product precipitated upon cooling to room temperature as colorless microcrystals. (12.5 mg, $11.3 \mu \mathrm{mol}, 45 \%$; Mp. $197^{\circ} \mathrm{C}$ (dec.)). Due to the low-solubility and the equilibrium of fac-1 and mer-1 in solution, reliable ${ }^{13} \mathrm{C}$ - and ${ }^{125} \mathrm{Te}-\mathrm{NMR}$ spectra could not be obtained.

fac-1: ${ }^{1} \mathrm{H}-\mathrm{NMR}\left(200.1 \mathrm{MHz}, \mathrm{CDCl}_{3}\right) \delta=7.99\left(\mathrm{~d},{ }^{3} \mathrm{~J}=7.9 \mathrm{~Hz}, 1 \mathrm{H}\right), 7.42(\mathrm{~m}, 18 \mathrm{H}), 7.18(\mathrm{dd}, J=7.9$, $3.4 \mathrm{~Hz}, 4 \mathrm{H}), 6.71\left(\mathrm{~d},{ }^{3} \mathrm{~J}=7.4 \mathrm{~Hz}, 1 \mathrm{H}\right), 6.22\left(\mathrm{~d},{ }^{3} \mathrm{~J}=7.5 \mathrm{~Hz}, 1 \mathrm{H}\right), 3.50(\mathrm{~s}, 4 \mathrm{H}), 3.45-3.15(\mathrm{~m}, 4 \mathrm{H}) \mathrm{ppm}$. ${ }^{31} \mathrm{P}-\mathrm{NMR}\left(81.0 \mathrm{MHz}, \mathrm{CDCl}_{3}\right) \delta=-0.9$ (s), -25.0 (s) ppm. ESI-MS $\left(\mathrm{CH}_{2} \mathrm{Cl}_{2} / \mathrm{CH}_{3} \mathrm{CN}\right.$ 1:10, positive mode): $m / z=1073.4\left(\mathrm{C}_{51} \mathrm{H}_{38} \mathrm{O}_{3} \mathrm{P}_{2} \mathrm{ReTe}\right)$ for $\left[\left(6-\mathrm{Ph}_{2} \mathrm{P}-\mathrm{Ace}-5-\right)_{2} \mathrm{Te}-\mathrm{Re}(\mathrm{CO})_{3}\right]^{+}$. FTIR: $\widetilde{v}(\mathrm{CO})=2018,1928,1882 \mathrm{~cm}^{-1}$. mer-1: ${ }^{31} \mathrm{P}-\mathrm{NMR}\left(81.0 \mathrm{MHz}, \mathrm{CDCl}_{3}\right) \delta=14.7\left(\mathrm{~d}, J\left({ }^{31} \mathrm{P}_{-}{ }^{31} \mathrm{P}\right)=11.6 \mathrm{~Hz}\right),-26.4\left(\mathrm{~d}, J\left({ }^{31} \mathrm{P}_{-}{ }^{31} \mathrm{P}\right)=11.6 \mathrm{~Hz}\right) \mathrm{ppm}$.

Synthesis of (6-Ph 2 P-Ace-5- $)_{2} \mathrm{TeMn}(\mathrm{CO})_{3} \mathrm{Br}$ (fac-2): $\mathrm{Mn}(\mathrm{CO})_{5} \mathrm{Br}(17.5 \mathrm{mg}, 64.4 \mu \mathrm{mol})$ was added to a solution of (6-Ph ${ }_{2} \mathrm{P}-$ Ace-5- $)_{2} \mathrm{Te}(50 \mathrm{mg}, 62.3 \mu \mathrm{mol})$ in THF $(10 \mathrm{~mL})$. The mixture was continually stirred at $65{ }^{\circ} \mathrm{C}$ for $32 \mathrm{~h}$. The product precipitated upon cooling to room temperature as orange microcrystals (30 mg, $29.4 \mu \mathrm{mol}, 47 \%$; Mp. $185^{\circ} \mathrm{C}$ (dec.)). Due to the low-solubility, the equilibrium between $f a c-2$ and mer-2 in solution and the concomitant formation of 3 reliable ${ }^{13} \mathrm{C}$ - and ${ }^{125} \mathrm{Te}-\mathrm{NMR}$ spectra could not be obtained.

fac-2: ${ }^{31} \mathrm{P}-\mathrm{NMR}\left(81.0 \mathrm{MHz}, \mathrm{CDCl}_{3}\right) \delta=50.4(\mathrm{~s}, \mathrm{br}), 39.9(\mathrm{~s}, \mathrm{br}),-27.4\left(\mathrm{~d}, J\left({ }^{31} \mathrm{P}-{ }^{31} \mathrm{P}\right)=7.2 \mathrm{~Hz}\right)$ ppm. mer-2: ${ }^{1} \mathrm{H}-\mathrm{NMR}\left(200.1 \mathrm{MHz}, \mathrm{CDCl}_{3}\right) \delta=7.95\left(\mathrm{~d},{ }^{3} \mathrm{~J}=7.2 \mathrm{~Hz}, 2 \mathrm{H}\right), 7.80-6.85(\mathrm{~m}, 26 \mathrm{H}), 3.41(\mathrm{~m}, 8 \mathrm{H}) \mathrm{ppm} .{ }^{31} \mathrm{P}-\mathrm{NMR}$ $\left(81.0 \mathrm{MHz}, \mathrm{CDCl}_{3}\right) \delta=50.0(\mathrm{~s}, \mathrm{br}),-27.9\left(\mathrm{~d}, J\left({ }^{31} \mathrm{P}-31 \mathrm{P}\right)=7.2 \mathrm{~Hz}\right)$ ppm. ESI-MS $\left(\mathrm{CH}_{2} \mathrm{Cl}_{2} / \mathrm{CH}_{3} \mathrm{CN}\right.$ 1:10, positive mode): $m / z=943.1\left(\mathrm{C}_{51} \mathrm{H}_{38} \mathrm{O}_{3} \mathrm{P}_{2} \mathrm{MnTe}\right)$ for $\left[\left(6-\mathrm{Ph}_{2} \mathrm{P}-\mathrm{Ace}-5-\right)_{2} \mathrm{Te}-\mathrm{Mn}(\mathrm{CO})_{3}\right]^{+}$. FTIR: $\widetilde{v}(\mathrm{CO})=2011$, $1939,1895 \mathrm{~cm}^{-1}$.

Synthesis of $\left(6-\mathrm{Ph}_{2} \mathrm{P}-\mathrm{Ace}-5-\right)_{2} \mathrm{TeMn}(\mathrm{CO})_{2} \mathrm{Br}(3) . \mathrm{Mn}(\mathrm{CO})_{5} \mathrm{Br}(7.00 \mathrm{mg}, 25.5 \mu \mathrm{mol})$ was added to a solution of (6- $\mathrm{Ph}_{2} \mathrm{P}$-Ace-5- $)_{2} \mathrm{Te}(20.0 \mathrm{mg}, 24.9 \mu \mathrm{mol})$ in THF $(5 \mathrm{~mL})$. The mixture was continually stirred at $65^{\circ} \mathrm{C}$ for $7 \mathrm{~d}$. The product precipitated upon cooling to room temperature as yellow microcrystals (10.0 mg, $10.0 \mu \mathrm{mol}, 40 \%$; Mp. $150{ }^{\circ} \mathrm{C}$ (dec.)). 
${ }^{1} \mathrm{H}-\mathrm{NMR}\left(360.3 \mathrm{MHz}, \mathrm{CD}_{2} \mathrm{Cl}_{2}\right) \delta=8.03\left(\mathrm{~s}, 4 \mathrm{H}, \mathrm{H}_{\mathrm{o}}\right), 7.60\left(\mathrm{~d}, J=7.1 \mathrm{~Hz}, 2 \mathrm{H}, \mathrm{H}_{4}\right), 7.52\left(\mathrm{~s}, 6 \mathrm{H}, \mathrm{H}_{\mathrm{m}+\mathrm{p}}\right)$, 7.35-7.19 (m, 8H, $\left.\mathrm{H}_{\mathrm{m}^{\prime}+\mathrm{p}^{\prime}}+\mathrm{H}_{8}\right), 7.19-7.05\left(\mathrm{~m}, 6 \mathrm{H}, \mathrm{H}_{\mathrm{o}^{\prime}}+\mathrm{H}_{4}\right), 7.00\left(\mathrm{~m}, 2 \mathrm{H}, \mathrm{H}_{7}\right), 3.50-3.34\left(\mathrm{~m}, 8 \mathrm{H}, \mathrm{H}_{1+2}\right)$. ${ }^{13} \mathrm{C}-\mathrm{NMR}\left(90.6 \mathrm{MHz}, \mathrm{CD}_{2} \mathrm{Cl}_{2}\right) \delta=150.8\left(\mathrm{~s}, \mathrm{C}_{\mathrm{c}}\right), 150.2\left(\mathrm{~s}, \mathrm{C}_{\mathrm{d}}\right), 139.9\left(\mathrm{t}, J=4.0 \mathrm{~Hz}, \mathrm{C}_{\mathrm{b}}\right), 139.0\left(\mathrm{~s}, \mathrm{C}_{4}\right), 136.8$ $\left(\mathrm{t}, J=5.1 \mathrm{~Hz}, \mathrm{C}_{\mathrm{a}}\right), 136.2\left(\mathrm{~s}, \mathrm{C}_{7}\right), 135.7\left(\mathrm{t}, J=18.1 \mathrm{~Hz}, \mathrm{C}_{\mathrm{i}}\right), 135.7\left(\mathrm{~d}, J=18.0 \mathrm{~Hz}, \mathrm{C}_{\mathrm{i}^{\prime}}\right), 134.5(\mathrm{t}, J=4.9 \mathrm{~Hz}$, $\left.\mathrm{C}_{\mathrm{o}}\right), 133.1\left(\mathrm{t}, J=4.6 \mathrm{~Hz}, \mathrm{C}_{\mathrm{o}^{\prime}}\right), 129.8\left(\mathrm{~s}, \mathrm{C}_{\mathrm{m}}\right), 129.0\left(\mathrm{~s}, \mathrm{C}_{\mathrm{m}^{\prime}}\right), 127.8\left(\mathrm{t}, J=4.4 \mathrm{~Hz}, \mathrm{C}_{\mathrm{p}}\right), 127.5(\mathrm{t}, J=4.2 \mathrm{~Hz}$, $\left.\mathrm{C}_{\mathrm{p}^{\prime}}\right), 127.0\left(\mathrm{~d}, J=17.5 \mathrm{~Hz}, \mathrm{C}_{6}\right), 119.5\left(\mathrm{t}, J=3.9 \mathrm{~Hz}, \mathrm{C}_{3}\right), 119.4\left(\mathrm{~s}, \mathrm{C}_{8}\right), 104.6\left(\mathrm{t}, J=7.0 \mathrm{~Hz}, \mathrm{C}_{5}\right), 29.8$ $\left(\mathrm{s}, \mathrm{C}_{1}\right), 29.7\left(\mathrm{~s}, \mathrm{C}_{2}\right) .{ }^{31} \mathrm{P}-\mathrm{NMR}\left(81.0 \mathrm{MHz}, \mathrm{CD}_{2} \mathrm{Cl}_{2}\right) \delta=59.7$ (s, br). ${ }^{125} \mathrm{Te}-\mathrm{NMR}\left(113.7 \mathrm{MHz}, \mathrm{CD}_{2} \mathrm{Cl}_{2}\right)$ $\delta=753.1$ (s). ESI-MS $\left(\mathrm{CH}_{2} \mathrm{Cl}_{2} / \mathrm{CH}_{3} \mathrm{CN} 1: 10\right.$, positive mode): $m / z=859.4\left(\mathrm{C}_{50} \mathrm{H}_{36} \mathrm{MnO}_{2} \mathrm{P}_{2} \mathrm{Te}\right)$ for [[(6-Ph $\left.\left.\left.{ }_{2} \mathrm{P}-\mathrm{Ace}-5-\right)_{2} \mathrm{Te}-\mathrm{Mn}(\mathrm{CO})_{2}\right]-2 \mathrm{CO}\right]^{+}$. FTIR: $\widetilde{v}(\mathrm{CO})=1936(\mathrm{~s}), 1852(\mathrm{~s}) \mathrm{cm}^{-1}$.

X-ray crystallography. Single crystals of $f a c-\mathbf{1}$, fac-2, and $\mathbf{3}$ were grown by slow evaporation of $\mathrm{CH}_{2} \mathrm{Cl}_{2}$ solutions that contained small amounts of $n$-hexane. Intensity data of $f a c-\mathbf{1}, f a c-2 \cdot \mathrm{CH}_{2} \mathrm{Cl}_{2}$, and $\mathbf{3} \cdot 2 \mathrm{CH}_{2} \mathrm{Cl}_{2}$ were collected at $100 \mathrm{~K}$ on a Bruker Venture D8 diffractometer with graphite-monochromated Mo-K $\alpha$ $(0.7107 \AA)$ radiation. All structures were solved by direct methods and refined based on $\mathrm{F}^{2}$ by use of the SHELX program package as implemented in WinGX [23]. Strongly disordered solvent molecules were accounted using the SQUEEZE routine for 1 and $\mathbf{2}$ [24]. All non-hydrogen atoms were refined using anisotropic displacement parameters. Hydrogen atoms attached to carbon atoms were included in geometrically calculated positions using a riding model. Crystal and refinement data are collected in Table 2.

Table 2. Crystal data and structure refinement of $f a c-\mathbf{1}, f a c-2$, and $\mathbf{3}$.

\begin{tabular}{|c|c|c|c|}
\hline & $f a c-1$ & fac-2 $\cdot \mathrm{CH}_{2} \mathrm{Cl}_{2}$ & $3 \cdot 2 \mathrm{CH}_{2} \mathrm{Cl}_{2}$ \\
\hline Formula & $\mathrm{C}_{51} \mathrm{H}_{36} \mathrm{ClO}_{3} \mathrm{P}_{2} \mathrm{ReTe}$ & $\mathrm{C}_{52} \mathrm{H}_{38} \mathrm{BrCl}_{2} \mathrm{MnO}_{3} \mathrm{P}_{2} \mathrm{Te}$ & $\mathrm{C}_{52} \mathrm{H}_{40} \mathrm{BrCl}_{4} \mathrm{MnO}_{2} \mathrm{P}_{2} \mathrm{Te}$ \\
\hline Formula weight, $\mathrm{g} \mathrm{mol}^{-1}$ & 1107.99 & 1106.11 & 1163.03 \\
\hline Crystal system & Triclinic & Monoclinic & Triclinic \\
\hline Crystal size, $\mathrm{mm}$ & $0.09 \times 0.08 \times 0.06$ & $0.10 \times 0.08 \times 0.05$ & $0.09 \times 0.08 \times 0.08$ \\
\hline Space group & $P \overline{1}$ & $P 2_{1} / n$ & $P \overline{1}$ \\
\hline$a, \AA$ & $12.2258(3)$ & $10.2073(2)$ & $12.542(5)$ \\
\hline$b, \AA$ & $14.3531(3)$ & $22.4724(5)$ & $12.738(5)$ \\
\hline$c, \AA$ & $14.8486(3)$ & $19.7629(5)$ & $15.516(5)$ \\
\hline$\alpha,{ }^{\circ}$ & $78.509(1)$ & 90 & $100.490(5)$ \\
\hline$\beta, \circ$ & 77.874(1) & 101.421(1) & $100.078(5)$ \\
\hline$\gamma^{\circ}$ & $89.216(1)$ & 90 & $100.930(5)$ \\
\hline$V, \AA^{3}$ & 2495.33(9) & $4443.5(2)$ & $2336(2)$ \\
\hline Z & 2 & 4 & 2 \\
\hline$\rho_{\text {calcd }}, \mathrm{Mg} \mathrm{m}^{-3}$ & 1.475 & 1.653 & 1.653 \\
\hline$\mu(\operatorname{Mo~K\alpha }), \mathrm{mm}^{-1}$ & 3.163 & 2.077 & 2.089 \\
\hline$F(000)$ & 1080 & 2200 & 1156 \\
\hline$\theta$ range, deg & 2.31 to 29.57 & 2.29 to 27.56 & 2.38 to 30.12 \\
\hline \multirow[t]{3}{*}{ Index ranges } & $-16 \leq h \leq 16$ & $-13 \leq h \leq 12$ & $-17 \leq \mathrm{h} \leq 17$ \\
\hline & $-19 \leq \mathrm{k} \leq 19$ & $-29 \leq \mathrm{k} \leq 29$ & $-17 \leq \mathrm{k} \leq 17$ \\
\hline & $-20 \leq 1 \leq 20$ & $-25 \leq 1 \leq 25$ & $-21 \leq 1 \leq 21$ \\
\hline No. of reflns collected & $27 \overline{5}, 62 \overline{26}$ & $12 \overline{20}, 248$ & $20 \overline{2}, 87 \overline{3}$ \\
\hline Completeness to $\theta_{\max }$ & $99.8 \%$ & $99.8 \%$ & $99.8 \%$ \\
\hline No. indep. Reflns & 13,983 & 10,262 & 13,719 \\
\hline No. obsd reflns with $(I>2 \sigma(I))$ & 13,702 & 8891 & 11,512 \\
\hline No. refined params & 532 & 553 & 556 \\
\hline $\operatorname{GooF}\left(F^{2}\right)$ & 1.074 & 1.042 & 1.054 \\
\hline$R_{1}(F)(I>2 \sigma(I))$ & 0.0148 & 0.0559 & 0.0553 \\
\hline$w R_{2}\left(F^{2}\right)$ (all data) & 0.0385 & 0.1690 & 0.1529 \\
\hline Largest diff peak/hole, e $\AA^{-3}$ & $0.844 /-0.846$ & $1.724 /-1.561$ & $1.955 /-1.665$ \\
\hline CCDC number & 1861602 & 1861603 & 1861604 \\
\hline
\end{tabular}

Figures 1-4 were created using DIAMOND [25]. Crystallographic data (excluding structure factors) for the structural analyses have been deposited with the Cambridge Crystallographic Data Centre. Copies of this information may be obtained free of charge from The Director, CCDC, 12 Union Road, Cambridge CB2 1EZ, UK (Fax: +44-1223-336033; e-mail: deposit@ccdc.cam.ac.uk). 
Supplementary Materials: The following are available online.

Author Contributions: T.G.D. carried out the experimental work, the spectroscopic characterization and participated in writing the manuscript. As Co-Investor, E.H. was involved in the interpretation of the results and also participated in writing the manuscript. As Crystallographer, E.L. carried out the X-ray structure analyses. As Principal Investigator, J.B. was responsible for writing the publication.

Acknowledgments: The Deutsche Forschungsgemeinschaft (DFG) is gratefully acknowledged for financial support.

Conflicts of Interest: The authors declare no competing financial interest.

\section{References}

1. Murray, S.G.; Hartley, F.R. Coordination chemistry of thioethers, selenoethers, and telluroethers in transition-metal complexes. Chem. Rev. 1981, 81, 365-414. [CrossRef]

2. Gysling, H.J. The ligand chemistry of tellurium. Coord. Chem. Rev. 1982, 42, 133-244. [CrossRef]

3. Singh, A.K.; Srivastava, V. The Chemistry of Multidentate Organotellurium Ligands. J. Coord. Chem. 1992, 27, 237-253. [CrossRef]

4. Hope, E.G.; Levason, W. Recent developments in the coordination chemistry of selenoether and telluroether ligands. Coord. Chem. Rev. 1993, 122, 109-170. [CrossRef]

5. Singh, A.K.; Sharma, S. Recent developments in the ligand chemistry of tellurium. Coord. Chem. Rev. 2000, 209, 49-98. [CrossRef]

6. Levason, W.; Orchard, S.D.; Reid, G. Recent developments in the chemistry of selenoethers and telluroethers. Coord. Chem. Rev. 2002, 225, 159-199. [CrossRef]

7. Torubaev, Y.; Pasynskii, A.; Mathur, P. Organotellurium halides: New ligands for transition metal complexes. Coord. Chem. Rev. 2012, 256, 709-721. [CrossRef]

8. Jain, V.K.; Chauhan, R.S. New vistas in the chemistry of platinum group metals with tellurium ligands. Coord. Chem. Rev. 2016, 306, 270-301. [CrossRef]

9. Gysling, H.J.; Luss, H.R. Synthesis and properties of the hybrid tellurium-phosphorus ligand phenyl o-(diphenylphosphino)phenyl telluride. X-ray structure of $\left[\mathrm{Pt}\left[\mathrm{PhTe}\left(\mathrm{o}-\left(\mathrm{PPh}_{2} \mathrm{C}_{6} \mathrm{H}_{4}\right)\right]_{2}\right]\left[\mathrm{Pt}(\mathrm{SCN})_{4}\right] \cdot 2 \mathrm{DMF}\right.$. Organometallics 1984, 3, 596-598. [CrossRef]

10. Kaur, R.; Singh, H.B.; Butcher, R.J. Syntheses and Characterization of Hybrid Bi- and Multidentate Tellurium Ligands Derived from N,N-Dimethylbenzylamine: Coordination Behavior of Bis[2-((dimethylamino)methyl) phenyl] Telluride with Chromium Pentacarbonyl. Organometallics 1995, 14, 4755-4763. [CrossRef]

11. Lin, T.P.; Gabbaï, F.P. Two-Electron Redox Chemistry at the Dinuclear Core of a TePt Platform: Chlorine Photoreductive Elimination and Isolation of a $\mathrm{Te}^{\mathrm{V}} \mathrm{Pt}^{\mathrm{I}}$ Complex. J. Am. Chem. Soc. 2012, 134, 12230-12238. [CrossRef] [PubMed]

12. Do, T.G.; Hupf, E.; Nordheider, A.; Lork, E.; Slawin, A.M.Z.; Makarov, S.G.; Ketkov, S.Y.; Mebs, S.; Woollins, J.D.; Beckmann, J. Intramolecularly Group 15 Stabilized Aryltellurenyl Halides and Triflates. Organometallics 2015, 34, 5341-5360. [CrossRef]

13. Kabir, S.E.; Ahmed, F.; Ghosh, S.; Hassan, M.R.; Islam, M.S.; Sharmin, A.; Tocher, D.A.; Haworth, D.T.; Lindeman, S.V.; Siddiquee, T.A.; et al. Reactions of rhenium and manganese carbonyl complexes with 1,8-bis(diphenylphosphino)naphthalene: Ligand chelation, $\mathrm{C}-\mathrm{H}$ and $\mathrm{C}-\mathrm{P}$ bond-cleavage reactions. J. Organomet. Chem. 2008, 693, 2657-2665. [CrossRef]

14. Hoyano, J.; Peterso, L.K. $\left[\mathrm{LM}(\mathrm{CO})_{3,4} \mathrm{X}\right]$ complexes of manganese(I) and rhenium(I) with phosphino- and thiophosphinatopyrazole ligands. Can. J. Chem. 1976, 54, 2697-2705. [CrossRef]

15. Hieber, W.; Kruck, T. Tellurorganyl-haltige Metallcarbonyle. Chem. Ber. 1962, 95, 2027-2041. [CrossRef]

16. Liaw, W.F.; Chiou, S.J.; Lee, W.Z.; Gene-Hsiang, P.; Peng, S.M. Syntheses and Characterization of Manganese-Tellurolate Complexes by Using Chelating Metalloligand cis-[Mn(Co $\left.)_{4}(\mathrm{TePh})_{2}\right]^{-}$and Potential Electrophilic TeMe ${ }^{+}$Reagent. J. Chin. Chem. Soc. 1996, 43, 29-35. [CrossRef]

17. Sadekov, I.D.; Uraev, A.I.; Garnovskii, A.D. Synthesis, reactions and structures of complexes of metal carbonyls and cyclopentadienyl carbonyls with organotellurium ligands. Russ. Chem. Rev. 1999, 68, 415-433. [CrossRef]

18. Lau, P.; Huttner, G.; Zsolnai, L. Trigonal-planar koordiniertes Selen bzw. Tellur in $\left[\mathrm{Cp}^{\prime}(\mathrm{CO})_{2} \mathrm{Mn}\right]_{2} \mathrm{EMes}^{+}$ $(\mathrm{E}=\mathrm{Se}, \mathrm{Te})$ : Verbindungen vom “Iniden"-Typ. J. Organometal. Chem. 1992, 440, 41-46. [CrossRef] 
19. Tondreau, A.M.; Boncella, J.M. The synthesis of PNP-supported low-spin nitro manganese(I) carbonyl complexes. Polyhedron 2016, 116, 96-104. [CrossRef]

20. Chen, X.; Femia, F.J.; Babich, J.W.; Zubieta, J. Synthesis, crystal structures and magnetic properties of transition metal-azide complexes coordinated with pyridyl nitronyl nitroxides. Inorg. Chim Acta 2001, 315, 147-152. [CrossRef]

21. Horikawa, H.; Horn, E.; Urushiyama, A.; Miyamoto, K. Crystal structure of tricarbonylbis [1,2-(diphenylphosphino)benzene]-manganese(I) bromide, $\mathrm{Mn}(\mathrm{CO})_{3}\left(\mathrm{C}_{30} \mathrm{H}_{24} \mathrm{P}_{2}\right) \mathrm{Br}$. Z. Kristallogr. New Cryst. Struct. 2010, 225, 687-688. [CrossRef]

22. Anderson, P.A.; Keene, F.R.; Horn, E.; Tiekink, E.R.T. Ambidentate coordination of the tripyridyl ligands 2,2':6' $2^{\prime \prime}$-terpyridyl, tris(2-pyridyl)-amine, tris(2-pyridyl)methane and tris(2-pyridyl)phosphine to carbonylrhenium centres: Structural and spectroscopic studies. Appl. Organomet. Chem. 1990, 4, 523-533. [CrossRef]

23. Farrugia, L.J. WinGX suite for small-molecule single-crystal crystallography. J. Appl. Cryst. 1999, 32, 837-838. [CrossRef]

24. Spek, A. Single-crystal structure validation with the program PLATON. J. Appl. Cryst. 2003, 36, 7-13. [CrossRef]

25. Brandenburg, K.; Putz, H. DIAMOND V3.1d; Crystal Impact GbR: Bonn, Germany, 2006.

Sample Availability: Samples of $f a c-\mathbf{1}, f a c-\mathbf{2}$, and $\mathbf{3}$ are available from the authors. 